\title{
Acute Presentation of Eventration of Diaphragm with Gastrothorax in an Infant
}

\author{
Faisal Basheer, * Qamar uz Zaman Khan, Jawad Jalil, Chaudhary Aqeel Safdar
}

Combined Military Hospital Rawalpindi, Pakistan

\begin{abstract}
Correspondence*: Faisal Basheer, Department of Pediatrics, $\mathrm{CMH}$, Rawalpindi, Pakistan
E-mail: fbasheer@gmail.com

Submitted: 12-07-2018

Conflict of Interest: None

(C) 2018, Basheer et al,

Accepted: 29-08-2018

Source of Support: Nil

This is an open-access article distributed under the terms of the Creative Commons Attribution License, which permits unrestricted use, distribution, and reproduction in any medium, provided the original work is properly cited.
\end{abstract}

\section{DEAR SIR}

Eventration of diaphragm is a congenital abnormality that results from failure of muscular development of either a part or whole of the hemi diaphragm. This results in abnormal structure and function of diaphragm leading to elevation of diaphragm with paradoxical movements.[1] Large eventration usually presents in neonatal period as it can cause mass effect in the thorax due to presence of stomach leading to ipsilateral lung collapse and mediastinal shift.[2] We present a case of a congenital diaphragmatic eventration (CDE) with a delayed presentation at 3 months of life with sudden onset severe respiratory failure requiring mechanical ventilation and emergency surgical correction.

A 3-month-old male infant, weighing $4.5 \mathrm{~kg}$ was referred from a peripheral centre with acute onset breathing difficulty, irritability, and poor feeding of two days duration. History of episodes of mild respiratory distress especially during feeding with cough was present. He also had occasional vomiting and was being treated as gastroesophageal reflux with pro-kinetic drugs. Previously done a 2-D ECHO was normal.Antenatal history revealed placental abruption in first trimester, gestational diabetes not requiring insulin, and oligohydramnios.

Baby was delivered at 36th weeks of gestation by elective caesarean section and weighed $3 \mathrm{~kg}$. Neonatal examination showed a healthy baby with no dysmorphism. Hypospadias with chordee was present. There was no NICU admission. Feeding was established soon after birth and the baby discharged within 24 hours after delivery.

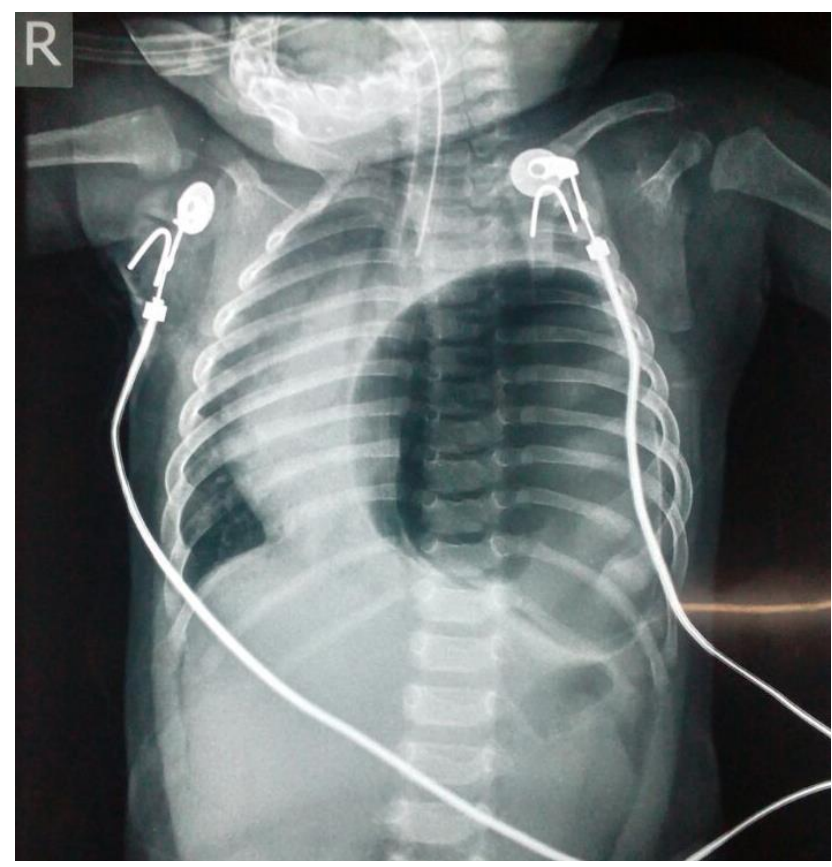

Figure 1: Chest $X$ ray showing an elevated left hemi-diaphragm with gastrothorax, collapsed left lung and mediastinal shift to right

On examination patient was irritable, with significant respiratory distress and marked intercostal and subcostal recessions. He was afebrile, with respiratory rate of 55 breaths per minute, pulse rate 170 beats per minute with oxygen saturation of 96 percent on $2 \mathrm{~L}$ by nasal 
prongs. Asymmetrical chest rise of left hemi-thorax, with shifting of trachea and apex beat to right were noted. On auscultation there were vesicular breath sounds on right side and left upper zone.

He was admitted in High Dependency Unit and nasogastric tube passed. Chest X-ray (CXR) showed displacement of left hemi diaphragm into thoracic cavity, collapsed left lung with mediastinal shift towards right and stomach occupying almost two third of left hemi thorax (Figure 1). A provisional diagnosis of CDE with gastrothorax was made. Baby's condition worsened at 6 hours of admission and shifted to Pediatric Intensive Care Unit. He was intubated and mechanical ventilation was started. High peak pressures were required to maintain arterial oxygenation above $60 \mathrm{mmHg}$. Considering difficult ventilation with risk of barotrauma, emergency corrective surgery planned after obtaining parental high-risk consent.

Plication of left hemi-diaphragm was done via an abdominal (left subcostal) approach. His postoperative recovery was smooth and uneventful. Postoperative $x$ ray showed complete expansion of left lung (Figure 2). $\mathrm{He}$ was extubated within 24 hours after surgery. His respiratory symptoms improved dramatically and feeding was started on 2 nd postoperative day. Patient was discharged on oral antibiotics on 4th postoperative day. At two weeks follow up he remained asymptomatic with weight gain of 300 grams. CDE is a rare diaphragmatic abnormality with a reported incidence of 1 in 2500-4000 live births predominantly in males.[3,4] Although CDE can present with respiratory distress but sudden onset respiratory distress without any signs of respiratory infection as seen in our patient is not a usual presentation.[5]

Abdominal organs especially stomach displaced into the thoracic cavity causes mass effect that can results in ipsilateral lung collapse and mediastinal shift as noted in our patient, resulting in respiratory distress. There may also be ipsilateral hypoplasia of lung.[2] Small defects do not produce symptoms while large defects can cause severe respiratory distress usually in neonatal period.[24]

It is very unusual for a large eventration to remain asymptomatic in early months of life as in our case and then presenting suddenly with respiratory difficulty.[4] CDE can be associated with various congenital defects; our patient had hypospadias with chordee. Severe respiratory distress requires mechanical ventilation and prompt surgical intervention as was the case in our patient.

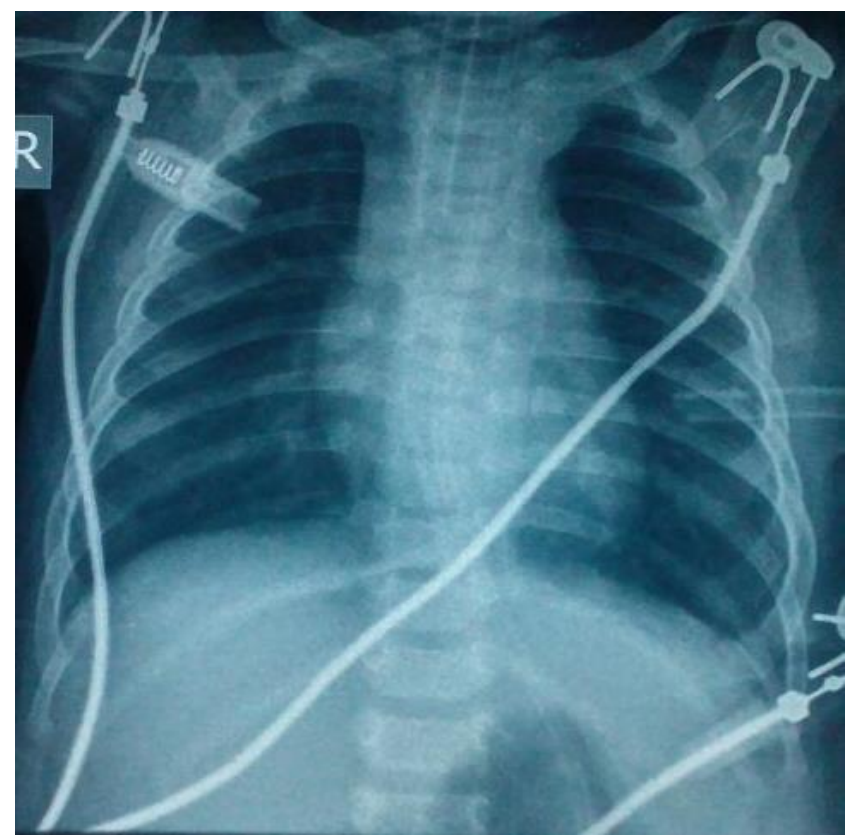

Figure 2: Postoperative $\mathrm{X}$ ray showing complete expansion of left lung

Consent: Authors declared that they have taken informed written consent, for publication of this report along with clinical photographs/material, from the legal guardian of the patient with an understanding that every effort will be made to conceal the identity of the patient however it cannot be guaranteed

Authors' Contribution: All authors contributed equally in concept, design, literature review, drafting the manuscript, and approval of the final manuscript.

\section{REFERENCES}

1. Mehrotra AK, Vaishnav K, Gupta PR, Khublani TK. Eventration of the right hemidiaphragm with multiple associations: A rare presentation. Lung India. 2014; 31:421.

2. Cilley RE, Coran A. Eventration of the diaphragm. Op Pediatr Surg. 2013;6.

3. Wu S, Zang N, Zhu J, Pan Z, Wu C. Congenital diaphragmatic eventration in children: 12years' experience with 177 cases in a single institution. J Pediatr Surg. 2015; 50:108892.

4. Makwana K, Pendse M. Complete eventration of right hemidiaphragm: A rare presentation. J Famil Med Prim Care. 2017; 6:870.

5. Alshorbagy A, Mubarak Y. Open transthoracic plication of the diaphragm for unilateral diaphragmatic eventration in infants and children. Korean $\mathrm{J}$ Thorac Cardiovasc Surg. 2015; 48:307. 